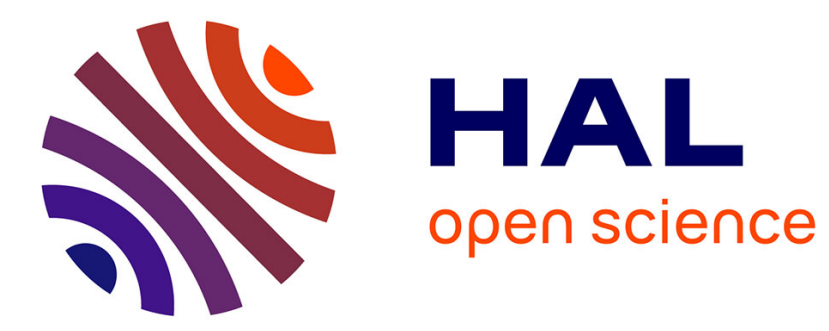

\title{
S'engager en situation de contrainte
}

\author{
Marie-Emmanuelle Pommerolle, Frédéric Vairel
}

\section{To cite this version:}

Marie-Emmanuelle Pommerolle, Frédéric Vairel. S'engager en situation de contrainte. Genèses. Sciences sociales et histoire, 2009, 77 (4), 10.3917/gen.077.0002 . hal-01648517

\section{HAL Id: hal-01648517 https://hal.science/hal-01648517}

Submitted on 26 Nov 2017

HAL is a multi-disciplinary open access archive for the deposit and dissemination of scientific research documents, whether they are published or not. The documents may come from teaching and research institutions in France or abroad, or from public or private research centers.
L'archive ouverte pluridisciplinaire HAL, est destinée au dépôt et à la diffusion de documents scientifiques de niveau recherche, publiés ou non, émanant des établissements d'enseignement et de recherche français ou étrangers, des laboratoires publics ou privés. 


\title{
S'ENGAGER EN SITUATION DE CONTRAINTE
}

Marie-Emmanuelle Pommerolle, Frédéric Vairel

\author{
Belin | « Genèses »
}

2009/4 $n^{\circ} 77 \mid$ pages 2 à 6

ISSN 1155-3219

ISBN 9782701153018

Article disponible en ligne à l'adresse :

https://www.cairn.info/revue-geneses-2009-4-page-2.htm

\section{Pour citer cet article :}

Marie-Emmanuelle Pommerolle, Frédéric Vairel « S'engager en situation de contrainte », Genèses 2009/4 (n 77), p. 2-6.

DOI 10.3917/gen.077.0002

Distribution électronique Cairn.info pour Belin.

(C) Belin. Tous droits réservés pour tous pays.

La reproduction ou représentation de cet article, notamment par photocopie, n'est autorisée que dans les limites des conditions générales d'utilisation du site ou, le cas échéant, des conditions générales de la licence souscrite par votre établissement. Toute autre reproduction ou représentation, en tout ou partie, sous quelque forme et de quelque manière que ce soit, est interdite sauf accord préalable et écrit de l'éditeur, en dehors des cas prévus par la législation en vigueur en France. Il est précisé que son stockage dans une base de données est également interdit. 


\section{S'engager en situation de contrainte}

près un développement rapide nourri d'échanges transatlantiques, la socio-
logie des mobilisations connaît une forme d'essoufflement conduisant cer-
tains auteurs à se tourner vers la production d'études sur des terrains autres que les espaces européens et nord-américains qui avaient servi de terreau à la majorité des travaux sur l'action collective ${ }^{1}$. L'objectif premier de ce décentrement géographique serait de «tester» les outils théoriques forgés dans des contextes démocratiques afin d'en valider les acquis et d'en percevoir les limites aux fins d'ajustement ${ }^{2}$. Les travaux présentés dans ce dossier, menés en Amérique latine (Angela Santamaria et Marie Laure Geoffray), au Maghreb (Montserrat Emperador), et dans le Golfe persique (Pascal Ménoret), vont plus loin que cela tout en ne répondant pas directement aux injonctions lancées depuis l'Europe ou les ÉtatsUnis, pour différentes raisons. À un premier niveau, l'apport de ces travaux est d'orienter le regard sociologique vers des objets trop peu explorés: l'internationalisation des mobilisations vue du Sud, et ses effets émancipateurs comme ses conditions inégalitaires, ou encore l'importance de l'évaluation du coût de l'action qui renvoie aux déterminants individuels de l'engagement. Ensuite, la nécessité de s'attirer la confiance des enquêtés dans des contextes où la sécurité des acteurs engagés comme celle de l'enquêteur est problématique, ou celle de les suivre dans leur campagne internationale obligent les chercheurs à mener des enquêtes ethnographiques longues et/ou répétées. Ces conditions nécessitent de multiplier les lieux d'observation sur le mode de l'anthropologie multisites et à s'interroger sur leurs rapports aux enquêtés, mais aussi sur la «vérité» scientifique et les conditions de sa restitution, quand certains résultats d'enquêtes ne sont pas dicibles pour des raisons politiques. Enfin, le décentrement par rapport aux questionnements désormais routiniers de la sociologie des mouvements sociaux emprunte deux directions. D'abord, l'objectif de ces recherches est de mieux comprendre, à travers des mobilisations collectives, la transformation de modes d'exercice du pouvoir. Ensuite, révélant l'expérimentation constante des pratiques de mobilisation, ces travaux s'interdisent de plaquer des outils théoriques sur des objets en constante redéfinition. Il ne s'agit surtout pas d'éclairer sous le soleil de nouveaux terrains des notions essoufflées par des usages aussi fréquents que peu critiques. Les catégories 
savantes, mais aussi les catégories pratiques comme celle de militantisme sont donc l'objet, dans les textes présentés, de toutes les précautions, sans être forcément celui de toutes les attentions.

Les différents textes font leur une avancée significative de la sociologie des mobilisations: l'attention portée aux pratiques protestataires ${ }^{3}$. La description en situation, la prise en compte des contextes et des temporalités de leur déploiement, l'intérêt pour les univers de sens dans lesquels elles s'insèrent ou des contraintes qui s'opposent à elles sont autant de pistes pour saisir dans le même mouvement l'activité des autorités et de leurs contestataires. Par-delà leur diversité, les pratiques militantes analysées ici ont en commun de nous éclairer sur les transformations concomitantes du pouvoir dans les espaces politiques dits «autoritaires» ou «en transition». L'action collective dans ces espaces, dont les contours sont marqués également par un nouveau rapport à l'économie globalisée, nous fait comprendre les pratiques de pouvoir de ces États et les perceptions qu'en ont les gouvernés. Dans cette perspective, les textes de ce dossier soulignent les capacités des États à négocier les modalités de leur domination quand légitimation et coercition ne sont pas des processus séparés mais s'entremêlent: ici, l'État cubain fait montre de capacités particulières à absorber les résistances; là, le cycle (irrégulier) manifestation-embauches ponctue les relations entre diplômés chômeurs et gouvernement marocain; plus loin, la monarchie saoudienne interdit tout militantisme des islamistes mais tolère l'expression électorale de leur mobilisation, en contraignant les formats de leur présence publique. Le code électoral qui régit les élections municipales empêche la constitution de listes et force les électeurs à voter à sept reprises dans autant de quartiers différents. Face à des électeurs privés des repères qu'offre la proximité ou l'affiliation politique, les activistes du Réveil islamique mobilisent le registre de l'hospitalité pour constituer leur électorat. En Colombie, l'exercice de la violence la plus crue s'inscrit dans des régimes de contrôle renouvelés, la délégation aux groupes paramilitaires de l'exercice de la coercition, parfois conforme aux intérêts de certaines entreprises étrangères, vient brouiller les cibles d'éventuelles actions protestataires.

Malgré ces interactions, l'incertitude - juridique, sociale, politique, économique - semble être au cœur de ces arrangements politiques, incertitude entretenue ou latente, mais toujours centrale pour comprendre les contraintes et les formes des mobilisations qui parviennent à s'y déployer. Face à une réaction imprévisible de la part de l'État, à des alliances fragiles, et à une opinion publique difficilement mobilisable, les pratiques protestataires observées sont en constante expérimentation. Les articles présentés cherchent avant tout à discerner les inflexions tactiques, les bifurcations, les retraits temporaires, et soulignent l'absence de routine mobilisatrice dans les cas étudiés. En ce sens, les contributeurs poursuivent certaines des notations les plus fécondes du courant de la mobilisation des ressources qui indiquent que l'action collective ne va jamais de soi. Observant les mobilisations «en train de se faire», ils s'appuient sur les concepts opératoires 
de l'analyse des mobilisations, tout en en faisant un usage distant, voire renversé. Difficile en effet d'affirmer l'existence d'un répertoire de l'action collective consolidé chez les intellectuels et artistes cubains, dont la trajectoire chaotique au sein d'un collectif qui oscille entre distribution de tracts clandestins et incorporation aux institutions étatiques, montre combien l'incertitude diffusée par le régime est déterminante dans les pratiques mobilisatrices. De même, derrière une banalisation affirmée par les observateurs, les manifestations de chômeurs marocains répondent, à chacune de leur occurrence, aux signaux lancés par l'État et ses forces de sécurité et s'y adaptent afin de minimiser les risques et les menaces qui pèsent sur la protestation. On assiste d'ailleurs à un retournement remarquable des intérêts de l'analyse «classique» de l'action collective. Les coûts de l'action, particulièrement la violence de l'État, informent les pratiques et les représentations liées aux mobilisations et prennent une place importante dans l'analyse des conditions de la mobilisation. En revanche la question des incitations collectives - la recherche de rétributions faisant généralement figure de passage obligé des explications du déclenchement de l'action collective - est mise de côté: soit elle n'est pas considérée comme déterminante, soit elle paraît si évidente pour expliquer le passage à l'action qu'elle n'appelle pas de développement particulier.

L'apport de ces travaux réside également dans l'attention portée à des questionnements habituellement périphériques de la sociologie des mobilisations. Si la transnationalisation de l'action collective a fait l'objet de nombreux ouvrages récents, ceux-ci pêchent par leur ethnocentrisme empirique ${ }^{4}$. À partir du portrait croisé de deux femmes colombiennes devenues militantes dans le cadre d'une campagne menée en Australie, il est possible d'observer combien le déploiement d'une cause à cheval sur plusieurs espaces de mobilisation est à la fois coûteux, concurrentiel, mais aussi porteur d'effets émancipateurs. Marquées par des ruptures biographiques répétées, ces femmes parviennent à se faire entendre dans un contexte affranchi des pesanteurs sociales et politiques de leur pays, quitte à jouer sur plusieurs registres de mobilisation, à entretenir des malentendus sur «la» cause défendue, à bricoler les faits. Ces usages de la «déconnexion» entre les espaces de mobilisation ne sauraient cependant masquer les inégalités et les formes de dépendance qui travaillent ces types de mobilisation, contrairement à certaines descriptions aseptisées de ces "réseaux» par la science politique nord-américaine ${ }^{5}$. La description minutieuse des acteurs des mobilisations, dans chacun des textes proposés, en droite ligne avec le renouveau des approches en termes de carrières, révèle aussi l'importance du coût individuel de l'action marquée par des risques considérables (exil, violences policières, attentats) mais jamais donnés une fois pour toutes.

Le surgissement de ces questions provient des méthodes d'enquêtes employées par les auteurs. Investigations longues, observations renouvelées, enquête risquée, on a là, en France au moins, la marque de fabrique d'une sociologie de l'action collective fondée sur les méthodes qualitatives ${ }^{6}$. La plus-value des enquêtes présentées 
ici est de s'interroger, parfois en creux, sur les modalités de restitution de pratiques officieuses ou risquées et sur les violences provoquant ou contraignant les engagements. Comment partager son savoir, en donner les sources, satisfaire aux exigences de «scientificité» et rendre public les résultats de l'enquête? Le cas colombien est significatif à cet égard: le «massacre de Tierra Bianca» que dénonce la campagne internationale est quasiment tu dans l'article, tout simplement car l'auteure n'est pas en mesure de produire des informations ou des interprétations fiables, objets de conflits politiques. Ici, le laboratoire international n'est pas seulement un havre qui éloignerait des fureurs du terrain. Ce déplacement est porteur d'effets sur l'écriture du compte rendu de sciences sociales. Afin d'éviter de réduire les «autochtones» concernés à de simples victimes, l'auteur-anthropologue - à l'instar du groupe enquêté qui prend le parti de se ressourcer à l'international - place cet événement à distance et privilégie l'action d'une porte-parole de ce groupe et de ses soutiens. Plutôt que de dire "ce qui s'est passé », l'objectif est de rendre compte de la construction conflictuelle de cette «cause». Comme à Cuba, l'autre méthode de mise à distance du politiquement sensible consiste à mener une ethnographie du très proche, en parcourant avec les acteurs les lieux les plus quotidiens de leur action. C'est en suivant les deux protagonistes colombiennes dans leurs activités de campagne et de sociabilité (réunions d'information comme soirée d'anniversaire) que l'auteure décèle les signes d'une intimité conflictuelle entre ces deux femmes et révèle les rapports sociaux qui sous-tendent ce militantisme extranational. À Cuba, en dépit de son délitement, l'appareil de surveillance contraint durement les acteurs et l'observateur. Il peut alors se muer en ressource: il oblige à suivre l'ensemble de relations sociales et de pouvoir dans lesquelles s'insèrent les collectifs d'intellectuels étudiés. L'une et l'autre de ces contributions placent leurs auteurs devant un dilemme des plus cruels: ici la protection de ceux qui sont devenus bien plus que de simples informateurs limite les ressources auxquelles le récit peut puiser pour affirmer sa véridicité. Lorsque l'écriture se rapproche des acteurs, l'auteur est face à l'arbitrage suivant: maintenir la fidélité la plus grande aux «données» et éviter de laisser des traces exploitables par les acteurs répressifs.

D'un cas à l'autre, les contraintes qui s'opposent aux acteurs ne coïncident pas nécessairement avec les dilemmes du social scientist, soucieux de protéger ses sources et de ne pas "griller» son terrain. L'enquête saoudienne dévoile toute la richesse d'une ethnographie de "demi-élections»; sa relative facilité semblable en cela au compte rendu des mobilisations marocaines, s'écarte de ce que l'on attendrait dans un tel contexte. Les contestataires sont des personnages publics, la parole aussi libérée chez les élites opposantes que chez les électeurs et l'enquêteur-photographe peut faire une tournée des bureaux de vote des plus paisibles. Contre-intuitive, cette situation souligne l'opacité de la notion même d'«autoritarisme». Le principal effet de cette contribution n'est pas de raffiner la définition que l'on pourrait donner de l'autoritarisme, pas davantage qu'elle ne permet de faire progresser ce qui en serait une «théorie». Au contraire, à l'instar des études sur Cuba et sur le Maroc, elle sou- 
ligne la faible consistance de la notion et son caractère peu opératoire lorsqu'on tente de lui faire dire plus que de caractériser des arrangements de pouvoir. A tel point que la «variable» répression, dont l'autoritarisme connote supposément un trop-plein, ne parvient pas à rapprocher les différents cas sous un type commun. Le régime de tolérance relâchée - mélange d'arbitraire, de flou dans les règles et de laisser faire bonhomme - qui organise les relations entre forces de sécurité et diplômés chômeurs au Maroc a peu en commun avec le formalisme des procédures électorales saoudiennes adossé à la criminalisation de toute forme d'opposition et aux tentatives permanentes de lui porter, physiquement, atteinte au nom de sa supposée «radicalisation». À Cuba, la contrainte sécuritaire est telle que l'agglutinement même des acteurs, la seule tentative d'agir de concert place sous la menace de la sanction. La force de la matrice révolutionnaire réside dans la possibilité de contrainte en profondeur des anticipations des acteurs qui osent s'en défier et dans la capacité de ce système d'emprise à tourner la contestation par des formes subtiles de canalisation, d'intégration, entre officialisation de l'informel et institutionnalisation de la critique. Poursuivre le voyage en Colombie ne sauverait guère cette entreprise de classement. La «transition» du régime s'est accompagnée d'une garantie plus effective de droits divers, au moins pour certains secteurs de la population, du recours régulier au suffrage universel pour désigner les détenteurs de charges publiques. Elle n'a pour autant guère pacifié de larges pans du territoire, contraignant leurs résidents à subir la violence ou l'exil.

\section{Marie-Emmanuelle Pommerolle et Frédéric Vairel}

\section{Notes}

1. Voir, par exemple, Charles Kurzman, «Structural Opportunities and Perceived Opportunities in Social-Movement Theory: Evidence from the Iranian Revolution of 1979 ", American Sociological Review, n 61, 1996, pp. 153-170.

2. C'est l'objectif des contributions rassemblées par Quintan Wiktorowicz dans Islamic Activism: a Social Movement Perspective, Bloomington, University of Indiana Press, 2004.

3. Voir Genèses, n 59, 2005, "Pratiques protestataires ». La récente actualité éditoriale confirme ce point: une collection des Presses de Sciences-po prête une attention particulière aux pratiques (grève, grève de la faim, usages contestataires des musiques urbaines); le Dictionnaire des mouvements sociaux (Olivier Fillieule, Lilian Mathieu et Cécile Péchu [éd.], Paris, Presses de Sciences-po, 2009) y consacre diverses notices (de barricade à violence contre soi en passant par émeutes, insurrection et pétition...).
4. Voir la synthèse critique qu'en fait Johanna Siméant dans: "Transnationalisation de l'action collective», in Éric Agrikoliansky, Olivier Fillieule et Isabelle Sommier, Manuel de sociologie des mouvements sociaux. Paris, La Découverte, 2009 (à paraître).

5. Sur ce point, voir Öngün Emre, «L'"effet retour" des stratégies transnationales. La modification de l'agenda et du répertoire d'action du syndicat KESK», in Gilles Dorronsoro (éd.), La Turquie conteste, Paris, CNRS, 2005, pp. 183-200. Voir plus généralement Yves Dezalay et Briant G. Garth, «Legitimating The New Legal Orthodoxy", in Y. Dezalay et B. G. Garth (éd.), Global Prescriptions. The Production, Exportation and Importation of a New Legal Orthodoxy, Ann Arbor, University of Michigan Press, 2002, pp. 306-334.

6. Olivier Grojean, «Investissement militant et violence contre soi au sein du Parti des travailleurs du Kurdistan », Cultures et conflits, n 63, 2006, pp. 101-112. 